\title{
Evolutions et perspectives de la strategie de Zara: en Amerique Latine
}

\section{Giovanny Fernando Benavides*}

Evalutions and perspectives of the Zara strategy in Latinamerica

Evolutions et perspectives de la strategie de Zara en Amerique Latine

Evaluçã̃ e perspectivas da estratégia de Zara na América Latina
* Magister en Negocios internacionales (Especialidad países europeos) Université de Pau et des pays de l'Adour; Especialista en Logística comercial nacional e internacional Universidad Forge Tadeo Lozano, Bogotá; Profesional en Comercio Internacional, Universidad Forge Tadeo Lozano, Bogotá 


\section{RESUMEN}

El grupo INDITEX se constituye como el número uno mundial de la industria textil, a pesar de la fuerte recesión en Europa. Para el último periodo del 2012, reporto un incremento en ventas del $22 \%$ respecto del mismo periodo en el año anterior, objeto de una estrategia innovadora de marcas y de una fuerte internacionalización la cual ha dado resultados significantes especialmente en Asia.
Palabras claves

Internacionalización

Externalización

Negocios

Empresas

Estrategia

Expansión

INDITEX

\section{ABSTRACT}

The aim of this paper is to make an analysis of the strategies used by the Inditex group, which allows it to reach a significant market positioning in Europe and also to analyze the internationalization strategies which apply to Latin America in its process of expansion, based on its largest market, Asia. For this reason, we initially evaluate the global market positioning of Zara as a brand name, focusing on a wide variety of perspectives (competition, development, sales, etc.), reflected on the European and Latin American markets, then stating all possible observations of the object of study, and finally highlighting its market penetration strategy potentials towards the Asian market.

\section{RESUMÉÉ}

L'objectif de cet article est double: analyser les stratégies mise en place par le groupe INDITEX lui ayant permis d'atteindre une position privilégiée en Europe et étudier son processus d'expansion et les stratégies d'internationalisation employées en Amérique latine. Nous commencerons pour cela à évaluer le positionnement global de la marque Zara (concurrence, développement, ventes, etc.), les perspectives des marchés européens et d'Amérique latine pour ensuite mettre en évidence les observations réalisées lors de cette étude renforçant le potentiel de la mise en place d'une stratégie similaire pour le marché asiatique.

\section{RESUMO}

Este artigo objetiva realizar uma análise das estratégias utilizadas pelo grupo INDITEX que lhe permitiram atingir uma posição significativa na Europa, e depois analisar as estratégias de internacionalização que foram aplicadas na América Latina durante seu processo de expansão baseados em seu maior mercado, a Asia. Para isto inicialmente foi avaliado o posicionamento global de Zara como marca desde as perspectivas (competência, desenvolvimento, vendas, etc.), a partir dos mercados europeus e da América Latina para depois propor as possíveis observações que são o objetivo deste estudo, ressaltando as potencialidades de uma estratégia de penetração no mercado asiático.

\section{Palavras-chave}

Internacionalização Externalização

Negócios

Empresas

Estratégia Expansão INDITEX 


\section{INTRODUCTION.}

$\mathbf{L}$ e groupe Inditex, et sa marque leader Zara, est l'un des leaders espagnols et européens du prêt-à-porter. Le groupe, au travers de ses différentes marques, s'est toujours prévalu d'une fidélité en termes de production à la ville de $\mathrm{La}$ Corogne; ville galicienne où Armancio Ortega a fondé sa première usine. De là, le groupe s'est étendu vers les villes du nord-ouest de la péninsule avant de s'implanter dans les grandes villes d'Espagne. Le groupe s'est depuis fortement internationalisé, et sa première implantation hors d'Europe s'est réalisée en Amérique Latine.

Ce marché a toujours été au centre de la stratégie de Zara, notamment en raison de sa proximité culturelle. Depuis lors, le groupe n'a cessé de se développer, principalement en Asie. Cependant, le marché du vêtement est devenu fortement compétitif, d'une part en raison d'une concurrence sur les produits e base en provenance des régions de production à bas coûts et d'autre part en raison de la concurrence d'entreprises mondiales sur des produits de gammes égales ou supérieures telles que $\mathrm{H} \& \mathrm{M}$ (Suède) et GAP (Etats-
Unis). La marque Zara, considérée comme du «Armani à prix raisonnables» selon une analyse de Goldman Sachs, est aujourd'hui présente dans 86 pays avec un réseau de plus de 1.763 boutiques situées dans les zones stratégiques des principales villes. Zara arrive donc au terme d'une phase de son développement.

Se pose désormais, pour le groupe Inditex, la question du renouvellement afin de redonner à la marque un nouveau souffle, surtout pour les pays où elle

est présente depuis plusieurs années. Alors qu'en Europe le modèle se comporte de façon équilibrée, les problèmes semblent surgir en Amérique Latine

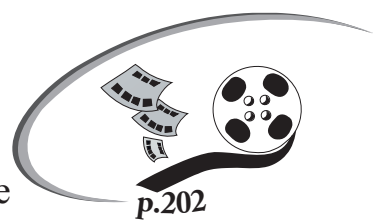
où les perspectives de développement commercial dépendent sensiblement de la stabilité économique des pays de la région ainsi que d'un modèle logistique performant devant résoudre les problèmes liés à un approvisionnement toujours plus onéreux. 


\section{UNE STRATEGIE D'EXTERNALISATION BASÉE SUR UN MODÈLE LOGISTIQUE PERFORMANT}

$\mathbf{P}$ our le groupe Inditex, la mise en évidence de ses origines a toujours été essentielle. Sa production et ses ventes ont pour cela été d'abord centrées en Galice, siège central de la société et poursuivies dans le reste de 1'Espagne puis au Portugal avec ses marques Zara (1975), Bershka (1998), Massimo Dutti (1995), Pull \& Bear (1991), Stradivarius (1999), Oysho (2001), Zara Home (2003), Uterque (2008), Kiddy Class (2002) (Skhuaban, pour des pays autres que l'Espagne et le Portugal) sans compter d'autres projets définis aux résultats négatifs (Belmiro Do Nascimento 2010). Zara étant à la fois le pionnier et la marque la plus reconnue du groupe, elle compte actuellement 1.121 magasins en Europe, et a commencé son processus d'internationalisation au Mexique en 1992, puis s'est tournée vers l'Amérique du Sud (1998) avec l'ouverture de Zara Argentine et Zara Venezuela. La dernière étape de cette internationalisation s'est effectuée en Amérique latine avec l'ouverture de ses premiers magasins au Pérou et en Equateur (2012). Aujourd'hui, le groupe dispose d'une stratégie de forte expansion en Asie avec une croissance orientée vers le marché chinois et ses 140 boutiques, représentant ainsi la deuxième présence commerciale après 1'Espagne.

Tableau 1. En Europe: une position de force

\begin{tabular}{|c|c|c|}
\hline Date de création & Pays d'implantation & Nombre de magasins \\
\hline 1975 & Espagne & 327 \\
\hline 1988 & Portugal & 62 \\
\hline 1990 & France & 127 \\
\hline 1993 & Grèce & 41 \\
\hline 1994 & $\begin{array}{l}\text { Belgique } \\
\text { Suède }\end{array}$ & $\begin{array}{c}27 \\
9\end{array}$ \\
\hline 1995 & Malte & 1 \\
\hline 1996 & Chypre & 5 \\
\hline 1997 & $\begin{array}{l}\text { Islande } \\
\text { Turquie }\end{array}$ & $\begin{array}{c}2 \\
32\end{array}$ \\
\hline 1998 & Royaume - Uni & 65 \\
\hline 1999 & $\begin{array}{l}\text { Allemagne } \\
\text { Pologne } \\
\text { Pays Bas } \\
\end{array}$ & $\begin{array}{l}69 \\
42 \\
23 \\
\end{array}$ \\
\hline 2000 & $\begin{array}{l}\text { Autriche } \\
\text { Danemark }\end{array}$ & $\begin{array}{c}12 \\
2\end{array}$ \\
\hline 2001 & $\begin{array}{l}\text { Italie } \\
\text { Luxemburg } \\
\text { République Tchèque }\end{array}$ & $\begin{array}{c}90 \\
2 \\
6 \\
\end{array}$ \\
\hline 2002 & $\begin{array}{l}\text { Finlande } \\
\text { Suisse }\end{array}$ & $\begin{array}{c}4 \\
10 \\
\end{array}$ \\
\hline 2003 & $\begin{array}{l}\text { Slovénie } \\
\text { Slovaquie } \\
\text { Russie }\end{array}$ & $\begin{array}{c}5 \\
3 \\
69\end{array}$ \\
\hline
\end{tabular}


Tableau 1. En Europe: une position de force (continuación)

\begin{tabular}{|c|l|c|}
\hline Date de création & Pays d'implantation & Nombre de magasins \\
\hline 2004 & Estonie & 2 \\
& Lettonie & 4 \\
& Roumanie & 20 \\
& Hongrie & 7 \\
\hline 2005 & Monaco & 1 \\
\hline 2006 & Serbie & 4 \\
& Chine & 140 \\
\hline 2007 & Croatie & 9 \\
\hline 2008 & Ukraine & 9 \\
& Monténégro & 2 \\
\hline & Honduras & 1 \\
\hline 2012 & Pérou & 1 \\
\hline
\end{tabular}

Source. Calculs des auteurs sur la base de données Inditex

Comme indiqué dans le tableau ci-dessus, Zara a commencé son processus d'internationalisation en 1988. Cette externalisation est renforcée par une présence dans plus de 86 pays, stratégie qui selon l'auteur Castellanos (2002) est basée sur un modèle vertical, à savoir une implantation directe dans le pays, et où seule la mise en place d'autres stratégies d'externalisation se réaliseront si les règles juridiques du pays obligent le groupe à adopter un modèle de gestion différent, dans ce cas Inditex choisira de poursuivre son expansion de trois manière différentes:

- l'implantation directe.

- Le joint-venture.

- La franchise.

L'implantation directe est réalisée par le biais de filiales du groupe Inditex. C' est le modèle utilisé dans la majorité des cas: à la clôture de l'exercice 2012, 86\% des boutiques du groupe étaient gérées de cette façon contre $14 \%$ en franchise. C'est uniquement dans certains cas que les différences culturelles et les risques associés à l'activité économique de certains pays (la taille du marché par exemple) dissuadent le groupe d'investir directement. La franchise est réalisée par un seul partenaire pour tout le pays. Il s'agit généralement d'un grand groupe national avec une trajectoire et une expérience solide dans la distribution de prêt-à-porter s'acquittant d'une taxe comprise entre 5\% et $10 \%$ des ventes et fournissant un accès complet aux services de l'entreprise : ressources humaines, formation et logistique, et ce sans frais supplémentaires pour Inditex (Belmiro Do Nascimento et al 2010).

Les joint-ventures appuyées par des membres locaux sont réalisées seulement dans les pays où les caractéristiques même de l'entreprise doivent pouvoir compter sur des avantages dérivés qui peuvent être représentés par une association dont les membres apporteraient une connaissance préalable du produit. Les joint-ventures permettent des synergies qui obligent une mise en commun de capitaux avec des partenaires locaux pour accéder à certains marchés. (Breuzard, Frometin, 2005). Les compagnies locales avec lesquelles Inditex a un accord de joint-venture en vigueur sont Otto Versand en Allemagne, Bigi au Japon et le Groupe Percassi en Italie.

Concernant le franchising, et selon des recherches faites par Belmiro Do Nascimento et al (2010) il existe un risque élevé dans les pays culturellement éloignés, avec des marchés trop restreints ou ayant de faibles prévisions de vente comme l'Arabie Saoudite, le Koweït, Andorre ou la Malaisie. Ces auteurs affirment également que pour Zara, les franchisés suivent le même modèle d'affaires que les filiales quand il s'agit des marchandises, de l'emplacement ou de la conception du magasin, de la logistique et des ressources humaines mais que la franchise est responsable des investissements d'actifs et du recrutement du personnel. 


\subsection{Organisation de la production: Une production et distribution centralisée en Espagne}

Alors que toute l'attention de l'industrie textile est tournée vers les délocalisations, Zara a implanté ses 19 usines en Espagne où les coûts de main-d'œuvre sont pourtant deux à trois fois plus élevés qu'au Moyen-Orient, en Afrique du Nord ou en Europe de l'Est. La marque fabrique ellemême plus de la moitié de ses articles, ceux étant le plus à la mode ou qui ne souffrent pas de délais de fabrication. Le reste, les produits dits "de base" sont sous-traités à plus de $70 \%$ en Europe. L'enseigne basée à La Corogne (Espagne) gère en direct 965 de ses 1121 magasins implantés en Europe, aux Etats-Unis, en Amérique du Sud ou au Japon. Près d'un million d'articles par semaine sont emballés, étiquetés par référence, taille, couleur et destination, et sont envoyés aux quatre coins de la planète. Le taux de rotation est très élevé et la distribution simplifiée.

L'entrepôt d'Arteixo (La Corogne) est littéralement vidé deux fois par semaine permettant ainsi de livrer très fréquemment l'ensemble des magasins Zara. Les boutiques n'ont donc pas besoin de stock. Le groupe espagnol effectue des tournées intersites afin de rééquilibrer les éventuels surstocks ou ruptures entre plusieurs magasins sur un même site, de sorte qu'aucun produit ne retourne jamais vers l'entrepôt en Espagne.

Le modèle d'affaire deZara est basé sur un système logistique centré sur différents points géographiques espagnols et selon des caractéristiques de distribution propres. Il existe une sous-traitance de la production pour réduire les coûts générés par une main d'œuvre onéreuse mais permettant de faible coûts de distribution sur les différents entrepôts de la péninsule. Cette gestion est appelée « centres de distribution différenciés ». Massimo Dutti et Stradivarius centralisent leur distribution à Barcelone en raison de leurs origines communes. Ils partagent cette plateforme logistique avec Bershka et Oysho, l'un à Tordera, l'autre à Sallent, les deux villes se trouvant à proximité de Barcelone.

Toute la production de Pull \& Bear est centralisée sur le site de Naron et le site de Tempe prend en charge chaussures et maroquinerie. Pour ce qui est de Zara, le centre de distribution de Meco, situé dans les environs de Madrid, près de la ville Saragosse, est chargé de toute la production de Zara Home et de Kiddy's Class. Ces sites stratégiques favorisent la logistique de par leur proximité géographique avec une plate-forme logistique aéroportuaire (Badia 2008).

\subsection{Facteurs clefs de succès: flexibilité et réactivité}

La stratégie adoptée par Zara implique une logistique sans faille et un équipement informatique performant dédié à la gestion automatique et quotidienne du réassortiment. Il existe une communication mondiale et en temps réel avec l'ensemble des magasins. Zara mise sur les circuits courts: l'entreprise peut ainsi multiplier et alimenter les mini- collections et autres gammes de vêtement, aussi nombreuses que variées, tout au long de l'année pour accroître la fréquentation des magasins. Cette politique de séries limitées permet également de réduire les risques, les stocks et les délais. Sa réactivité tient donc à une chaîne logistique parfaitement optimisée : le cas Zara est l'exemple parfait d'une entreprise qui a su se différencier durablement de ses concurrents grâce à la puissance de sa «supply chain» (Schéma 1).

\section{Schéma 1- Intégration et réactivité de la chaîne logistique}

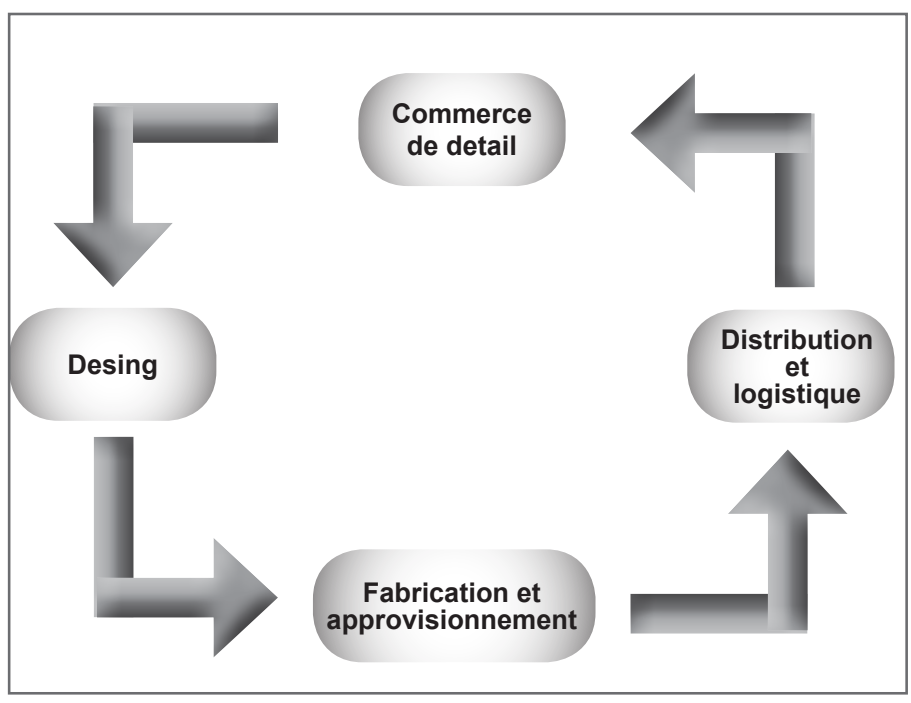

Source. Élaboration personnelle 
La plate-forme logistique conçoit et livre une nouvelle collection en 15 jours (design, fabrication et livraison) alors qu'il faut entre 3 et 4 mois à ses concurrents (Gap, H\&M) pour lancer une nouvelle collection sur le marché. La création de l'événement et de la rareté par la multiplicité de l'offre et son renouvellement fréquent « oblige » les clients à revenir régulièrement pour découvrir les nouveautés. La clientèle a la certitude de trouver chez Zara les derniers articles à la mode, adaptés aux soubresauts climatiques... Un avantage concurrentiel considérable dans un secteur fortement marqué par les aléas saisonniers.

Il faut ajouter que cette intégration, liée à la réduction des cycles de production, est intégrée à un système d'information optimisé géré par intranet et permettant une communication immédiate entre tous les acteurs de la chaîne:

- Remontée d'information des magasins (chiffres de ventes, remarques des clients,...); ce qui permet une livraison rapide, des échanges entre magasins et la création de modèles répondant aux exigences des clients.

- Les unités de production sont en relation directe avec les créateurs.
- Les fournisseurs ou sous -traitants sont « connectés » à l'entreprise.

Il est fondamental pour un groupe comme Inditex d'éviter au maximum les coûts de transfert avec des intermédiaires, sous-traitants ou franchisés qui pourraient opposer des forces de résistance à la fluidité du système. De ce fait, la distribution simplifiée est un des autres atouts de la chaîne logistique. Grâce à son système de production centralisé, Zara livre pratiquement tous ses magasins directement depuis la Corogne.

L'enseigne limite ainsi les niveaux intermédiaires, réduisant les stocks incompressibles et les délais. Que ce soit par camion ou par avion, la chaîne met entre 24 et 48 heures pour atteindre n'importe quel point de vente. Cette stratégie demande une connexion permanente avec les transitaires et les transporteurs qui permettent de répondre aux besoins logistiques de livraison.

Malgré une connexion optimum entre les différents modes de transport, la plupart des marchandises voyage toujours en camion. Ce mode de transports couvre en effet $80 \%$ des besoins de livraison, le reste des marchandises étant transportées par voie aérienne pour des raisons de délais de livraison et d'expédition. Le fret maritime et la voie ferrée ne sont pas utilisés (Badia, 2008). 


\section{EN AMERIQUE LATINE: UNE POSITION À RENFORCER}

\subsection{Développement sur des marchés aux comportements similaires}

Au début des années 1990, Zara et le groupe Inditex, alors en plein expansion, décident de se lancer sur le marché Sud-Américain. Après avoir analysé les coûts d'opportunité que cela pouvait représenter, Armancio Ortegua choisit comme stratégie initiale de se focaliser sur les pays Latinos. On peut se demander pourquoi orienter l'entreprise vers les pays sud-américains avant le reste de l'Europe ? Cela peut s'expliquer par une certaine proximité culturelle. En effet, même si les pays d'Amérique latine se sont «américanisés», ils restent proches de l'Espagne sur certains points (langues, croyances, traditions, etc.), et cela peut être identifié comme du marketing international basé sur un environnement culturel qui contient des variables incontrôlables. Certains auteurs comme Kim Lee (2005) définissent que les variables culturelles comme la religion, la langue, les valeurs et les attitudes, augmentent les probabilités de succès pour une entreprise qui partage ces valeurs. Cela dépend de plus d'une sensibilisation culturelle du pays où l'entreprise pense s'implanter et cela, Mr Ortegua semble l'avoir compris.

Après avoir analysé ces facteurs, Zara a donc estimé que le potentiel de développement était plus fort en Amérique Latine que dans les autres pays européens. Une segmentation similaire révèle que les pays LatinoAméricains peuvent compter sur une classe moyenne relativement importante, possédant un revenu disponible assez élevé (malgré un fort niveau d'endettement). Cette classe moyenne constitue une aubaine pour Zara qui en a fait son cœur de cible. D'un point de vue marketing, cette segmentation est tout à fait conforme au positionnement de Zara qui tend à cibler d'une part une clientèle avec revenu moyen acceptable-plutôtjeune, dynamique (segmentation par le revenu) et d'autre part une clientèle «branchée » plutôt hédoniste / chic qui attache de l'importance à l'apparence et qui souhaite se vêtir en harmonie avec son style de vie (segmentation psychographique). Les effets d'imitation sont également capitaux (imitation d'une «classe sociale plus élevée», «chic», extrêmement restreinte) et son impact sur l'imaginaire d'une société sont

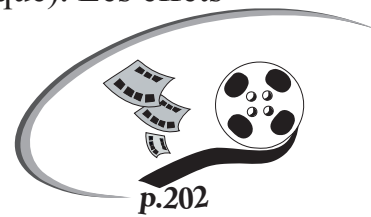
fort (réussite, style, occidentalisation forte,...). (Kotler et Armstrong, 2007)

Cette segmentation, pour la période considérée, était présente dans un pays comme le Mexique qui a été retenu pour le lancement de Zara comme test pilote d'internationalisation. D'autres magasins du groupe Inditex ont suivi cette stratégie dans un second temps. Les raisons du choix du Mexique sont multiples:

- Proximité des Etats-Unis et d'un modèle de consommation occidental (implantation aux Etats-Unis depuis 1989).

- Niveau de développement urbain et potentiel de consommation élevé (surtout à Mexico D.F).

En 1992; le premier magasin Zara ouvre dans la ville de Mexico. Le Mexique devient ainsi le 5ème pays d'implantation pour Zara après l'Espagne, le Portugal, les Etats-Unis et la France. Actuellement, c'est un des pays où le développement de l'entreprise est très important. En effet, le groupe Inditex y implanté la plupart de ses autres sociétés (Berksha, Pull \& Bear, Massimo Dutti, Zara Home, etc.), et le Mexique se place en 3ème position (derrière l'Espagne, le Portugal et la France) pour le nombre total d'enseignes présentes (245 magasins).

«Le Mexique a été le premier pays dans lequel le groupe a développé son modèle d'internationalisation complet, avant il était présent à New York et Paris, mais on ne peut pas vraiment parler d'une internationalisation jusqu'à ce qu'il ait atteint le Mexique », a déclaré Jésus Echevarria, directeur générale de communication et des relations institutionnelles d'Inditex à CNNexpacion.com. 


\subsection{Implantation «Européenne» Amérique Latine}

Après ce premier lancement positif, Zara décide d'aller vers d'autres pays latino-américains à fort potentiel. Après s'être lancé sur des marchés aussi surprenant que la Turquie, Israël ou encore le Japon, Inditex lance ses premiers magasins Zara en Argentine et au Venezuela en 1998. Ce « saut» vers de nouveaux pays ouvre une nouvelle phase d'implantation de Zara en Amérique Latine. En effet, l'entreprise a déjà dans ses cartons les projets d'implantation dans de nouveaux pays. Comme analysé précédemment, l'avantage pour Zara réside dans le fait que les collections présentées dans les pays Latino-américains sont similaires à celles qui se créent et se présentent en Europe. En général, les vêtements sont davantage «estivaux» (du fait que le climat est globalement plus chaud en Amérique Latine).

Après l'Argentine et du Venezuela, Inditex se lance simultanément, en 1999, dans 3 pays Latino-américains : Le Brésil, le Chili et l'Uruguay. Cette nouvelle vague d'implantation marque un véritable tournant pour l'entreprise qui montre clairement sa volonté de s'ancrer de plein pied dans la région et de rayonner dans la totalité du sous-continent.

Selon Diego Copado Fernández, Directeur de la Communication Corporate du Groupe Inditex «la croissance en Amérique Latine a été très forte dès le début des années 1990, et elle a continué à croître grâce à la multiplication des implantations mais également à la variété et l'amplitude de son offre de produits. L'Amérique Latine continue d'être considérée comme la seconde zone la plus importante pour le groupe, notamment en raison du nombre de magasins présents. " et ce malgré une forte croissance de l'Asie, Chine en tête.

Dernière phase d'implantation en Amérique Latine, Inditex décide de se lancer vers des pays moins «européanisés », et de façon générale, beaucoup plus petits en termes de marché que sont les pays d'Amérique centrale. Le processus d'implantation d'Inditex se terminera dans cette zone géographique avec les pays andins que sont la Colombie, le Pérou et l'Equateur, qui n'offraient pas les garanties suffisantes en terme de « risque pays » à la venue de capitaux étrangers, mais qui représentent un marché attrayant. (Tableau 2)
Tableau 2. Amérique Latine: Externalisation progressive

\begin{tabular}{|c|c|c|}
\hline Année & Pays d'implntation & $\begin{array}{c}\text { Nombre de } \\
\text { boutiques }\end{array}$ \\
\hline 1992 & Mexique & 56 \\
\hline 1998 & Argentine & 10 \\
& Venezuela & 9 \\
\hline 1999 & Brésil & 39 \\
& Chili & 8 \\
& Uruguay & 2 \\
\hline 2002 & El Salvador & 2 \\
& République Dominicaine & 3 \\
\hline 2004 & Panama & 2 \\
\hline 2005 & Costa Rica & 2 \\
\hline 2007 & Guatemala & 2 \\
& Colombie & 2 \\
\hline 2008 & Honduras & 1 \\
\hline 2012 & Pérou & 1 \\
\hline
\end{tabular}

Source. Calculs des auteurs sur la base de données Inditex

\subsection{Faire face aux instabilités et relever les défis de la région}

Le panorama Latino-Américain révèle une certaine division politique et économique avec des pays ouvert aux investissements étrangers : la Colombie, le Mexique, le Pérou, le Brésil, le Chili, le Costa Rica et des pays avec des caractéristiques protectionnistes comme le Venezuela, la Bolivie, l'Équateur, le Nicaragua et l'Argentine. Cet éventail des possibles offre un aperçu assez négatif souligné par des nationalisations au Venezuela, des résiliations de projets d'infrastructure en Bolivie, des poursuites judiciaires pour violations aux contrats en Equateur et des manifestations sociales qui paralysent les plans d'exploitation minière au Pérou laissant une image complexe du continent (America Economica, 2012).

D'après les dernières prévisions élaborées par Inditex, l'Amérique Latine est structurellement appelée à perdre de l'importance dans la composition du Chiffre d'Affaires (CA) de Zara. Ceci peut s'expliquer par trois facteurs majeurs:

- La perte de compétitivité-prix due aux fluctuations monétaires. 
- Les différences politiques régionales qui ne permettent pas une croissance homogène entre les pays.

- L'ouverture rapide de nouveaux magasins dans d'autres zones du monde.

Ce dernier élément constitue le point d'achoppement qui entraîne une moindre pondération de Zara Amérique Latine dans le résultat de Zara Monde (de façon moins marquée par Inditex qui dispose de l'éventail très large au Mexique de toutes les boutiques se trouvant dans le giron du groupe).

Zara se lance par ailleurs à l'assaut du marché asiatique, un marché prometteur aux vues des immenses agglomérations urbaines et de la présence d'une classe moyenne au pouvoir d'achat conséquent qui pourrait laisser envisager au groupe de changer sa politique d'internationalisation et de la remplacée par une politique de réductions des coûts de production en Asie qui se traduirait par une diminution du coût final du produit.

Cependant, l'objectif de Zara à moyen terme n'est pas de laisser 1'Amérique Latine à un second plan car la région représente la deuxième zone en terme de présence commerciale après l'Europe, et es potentiels de croissance sont encore immenses. Afin de mieux définir quels peuvent être les enjeux actuels pour Zara, on peut se pencher sur l'organisation du groupe dans la région.

\subsection{Des structures organisatrices cohérentes avec le terrain et la philosophie d'Inditex en Amérique Latine}

Al'image de sa maîtrise de la production, Zara, et le groupe Inditex, ont toujours voulu avoir le contrôle complet de l'intégralité du processus, depuis la création des collections à la vente au client final. En effet, Inditex dispose d'un degré d'intégration rarement égalé dans le domaine. Cette organisation globale mais coûteuse, lui permet néanmoins de réaliser de nouveau modèle de vêtements et surtout de les produire et de les expédier en un temps record et ce grâce un réseau de sous-traitants permettant au groupe de développer un modèle de production en réseau inter-connectés où un grand nombre de petites et moyennes entreprises sont chargées de la production, de la distribution et de la vente et avec lesquelles la maison mère maintien des liens basés sur l'externalisation et le modèle de franchise (Barreiro, 2008).

Au niveau de l'organisation commerciale, Zara évite au maximum l'attribution de franchise au profit de la création de filiale. Cette solution se révèle très demandeuse en capital, mais est la seule alternative pour un groupe souhaitant avoir une maîtrise complète du processus. La majorité des boutiques sont propriété de Zara. Seuls les petits pays en termes de taille ont opté pour la franchise. Il est néanmoins important de noter que les franchises ont été distribuées, non pas à des particuliers, mais à de grands groupes industriels possédant une expérience de la distribution. Le modèle de franchise est l'apanage de pays comme la République Dominicaine et le Salvador.

Le marché latino-américain se caractérise donc par une forte concentration urbaine, mais également par une absence de loi restreignant la surface allouée aux enseignes. Un autre atout des pays d'Amérique Latine est que leurs économies sont parmi les plus ouvertes du monde en ce qui concerne les investissements étrangers. Les formalités d'enregistrement des sociétés sont simples. Le décret 1853 du 8 septembre 1993 établit en Argentine autorise par exemple la même procédure administrative pour les investissements étrangers que pour les investissements locaux. Les politiques de nationalisation du gouvernement de Cristina Fernandez ne touchent pas encore le secteur textile et autorise le libre rapatriement des bénéfices vers la maison mère et la suppression des autorisations préalables à l'implantation. D'autre part, les accords douaniers entre l'Union Européenne et le Mercosur, la Colombie, le Pérou ou le Mexique permettent de proposer certains produits dans les deux zones, facilitant ainsi la réalisation d'économies d'échelle.

D'autre part, le coût de la main d'œuvre, dans une optique de production reste relativement faible. Il est tout à fait possible, comme le font les concurrents de Zara, de soustraiter une partie de la production dans des usines déjà présentes dans la région. On peut noter que des pays comme le Mexique présente des systèmes de «maquiladoras » ou que la Colombie offre une production textile hautement spécialisée, ce qui pourrait être une alternative intéressante pour Inditex dans le cadre d'un contexte de production. Cela peut être pertinent si l'on considère la forte 
implantation dans les deux pays. Toujours dans l'optique d'une implantation industrielle, l'hypothèse de la création d'une «division productive » prend tout son sens avec la perspective d'un rayonnement sur l'Amérique du Nord et l'Amérique latine.

La conception " intégrale » de Zara a été jusqu'à présent plutôt bénéfique alors qu'Inditex ouvre de nouvelles entités partout dans le monde, ce modèle est peut-être en train de connaître ses limites. Nous notons en effet certaines limites au modèle :

- Limites commerciales : La grande majorité des centres urbains d'importance possèdent une enseigne Zara et les autres grandes villes Latino-américaines ne possèdent pas une population suffisante pour permettre à Zara d'ouvrir de nouveaux magasins.

- Limites techniques/logistiques: Il devient difficile d'approvisionner un maximum de boutique sur un continent si dispersé et il devient donc essentiel de réévaluer le modèle logistique.

A cela s'ajoute le fait que pour Zara, le marché européen reste prioritaire, et que le groupe a également des visées sur l'Asie (bien que ceci puisse éventuellement aller en faveur de l'Amérique latine). Il est cependant nécessaire de développer une nouvelle approche pour l'Amérique Latine.

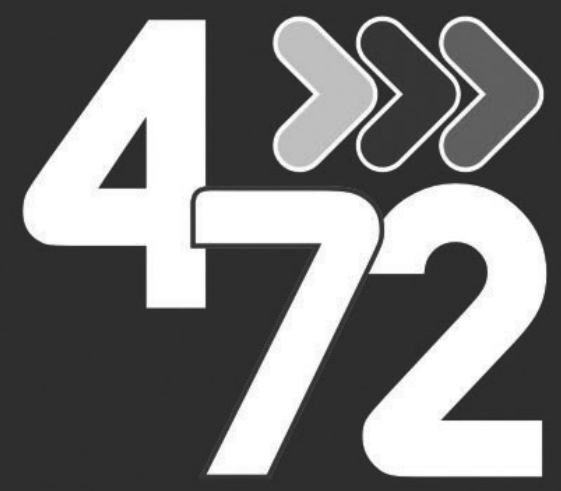

LA RED POSTAL DE COLOMBIA

w w w $4-72 \cdot c 0 m \cdot c 0$ 


\section{UNE NOUVELLE APPROCHE POUR L'AMERIQUE LATINE: STRATÉGIES D'IMPLANTATION}

D ans les pays riches, le produit industriel est de moins en moins un produit physique et de plus en plus un produit lié à une image, ce qui est en soi une innovation. Exception faite des textiles techniques (utilisés par exemple dans l'automobile ou l'aéronautique), il est devenu difficile d'innover en matière de production physique dans le textile- habillement. En revanche, la clé de l'innovation, dans ce secteur, est de faire changer les goûts, de créer une symbolique autour du vêtement. En conséquence, rien n'empêche de faire fabriquer la mode ailleurs. Et compte tenu des coûts de main-d'œuvre et des facilités de communication, cela même devenu beaucoup plus intéressant, le différentiel des salaires modifiant ainsi la localisation de la production.

Décathlon, groupe français qui exerce ses métiers de production d'articles de sport et de distributeur sur les 5 continents est présent au Mexique ou il réalise une activité de production. Il a par ailleurs démarré un type d'activité similaire en 1999 au Brésil et l'ouverture du premier magasin à Campinas en 2001. D'autre part, le suédois $\mathrm{H} \& \mathrm{M}$ a adopté une stratégie claire : avoir une marque forte mais produire à l'échelle régionale, en délocalisant au Mexique et dans les Caraïbes.

Carrefour a choisi la souplesse par rapport au cycle économique de chaque pays. En effet, le Brésil et l'Argentine ont traversé régulièrement des périodes d'hyper-inflation qui ont pu rendre délicate la gestion de magasins à grand nombre de références. La réponse de Carrefour a donc reposé sur une offre commerciale restreinte, concentrée sur des produits à rotation rapide. L'innovation chez Carrefour est constante avec par exemple de nouveaux produits et services apparaissant chaque année dans les magasins : les loisirs (vacances, spectacles), les produits-bio en France, le montage de pneus, les boutiques, les stations essence.

En exportant son concept d'hypermarché vers des pays en développement comme le Brésil ou l'Argentine à partir des années 70 , le groupe prenait le pari de l'adaptation du

format à des modes de

consommation «différents»

de ceux de l'Europe et dès le

début, cette adaptation a fonctionné

et la formule du «tout sous le même

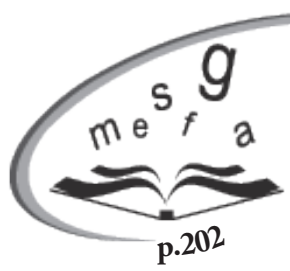

toit» dans des magasins à dominante alimentaire fait désormais partie intégrante du paysage commercial en Amérique Latine.

Nous considérons, en accord de différentes études de cas menées sur la stratégie Zara, que la politique logistique mise en place par le groupe est l'un des facteurs clés du succès obtenu. On peut identifier certaines de ces stratégies sur la base des exemples ci-dessus et des particularités de la région. Le modèle devrait varier dans le sens où la logistique, la production, la distribution et les ventes se rapprochent du consommateur final. En ce sens elles offrent les alternatives de mise en œuvre suivantes:

\subsection{Filiale industrielle}

Zara peut choisir de créer une unité de production complète et indépendante d'une implantation commerciale : il s'agit alors d'une délocalisation de production afin de profiter de différences de coût de main d'œuvre, d'un savoir-faire local ou encore d'une rationalisation de la logistique et de la distribution internationale. Même si la productivité du travail n'est pas toujours très élevée, la différence de salaire permet une diminution des coûts de production : cela permettrait à la société d'améliorer ses marges.

Les limites:

- Coût, rigidité actuelle.

- Recrutement du personnel.

- Formation à la politique d'Inditex, (formation aussi bien en Espagne que dans d'autres pays où la marque est implantée. A titre d'exemple, ne serait-ce que pour 
l'implantation commerciale de Zara au Maroc, le coût de la formation avoisine à ce jour plus de deux millions de $\mathrm{DH})$.

- Respect de la législation locale.

\subsection{Investissement en joint-venture}

Zara peut choisir de créer une société en commun avec une entreprise locale, et détiendrait dans ce cas une partie du capital. L'investissement dans une unité de production entraîne des coûts importants qu'il vaut mieux partager avec une entreprise locale qui connait bien son marché, qui dispose d'infrastructures et à qui l'exportateur apporte un savoir- faire technique et commercial intéressant.

Les limites:

- Contrôle de l'activité de la joint-venture, risque de mésentente sur le management stratégique, la répartition des bénéfices, perte du savoir- faire, etc.

\section{Une acquisition}

Au lieu de créer une filiale de toutes pièces (croissance interne), il peut être intéressant d'acquérir une entreprise existante (croissance externe). De gros avantages peuvent en résulter: gain de temps de mise en marche du processus de fabrication.

\section{Les limites}

- Recherche de synergies entre les activités des deux entreprises; imposer le modèle de Zara au modèle existant (formation du personnel).

- Abandon de la politique de contrôle stratégique.

\section{Localisation géographique}

Le Brésil possède un atout incomparable grâce à son offre insuffisante de coton pour un marché textile industrialisé. Le Brésil est le pays le plus grand et le plus industrialisé du MERCOSUR qui incorpore à son tissu industriel toutes les activités du secteur textile. Le pays produit des fibres naturelles et artificielles. La principale fibre naturelle est le coton - de mauvaise qualité -, mais aussi la soie, le « yute », le «ramio », et une production de laine.

Le Brésil dispose d'usines de production de tout type de fibres artificielles et synthétiques : nylon, polyester, acrylique, fibres oleophyniques, dérivés d'élastomère, etc. Les fibres sont transformées dans des filatures et ateliers de tissage. Dans l'habillement, le Brésil produit une grande variété de produits (tee-shirt, vêtements féminins, etc.) Les principaux domaines sont : les vêtements de prêt-àporter (environ $40 \%$ des ventes), vêtements de travail et de sécurité $(23 \%)$, textiles pour usage industriel et technique $(16 \%)$, vêtements classiques (12\%), et le linge de maison $(6 \%)$.

Le Brésil possède des industries productrices de produits chimiques (pigments, colorants), des machines et outils de travail spécialisés dans l'industrie. L'ensemble de la production de fibres est consommé par l'industrie brésilienne qui complète son approvisionnement avec l'importation de coton.

\section{Tableau 3. Brésil - Forces et faiblesses pour la production textile}

\begin{tabular}{|l|l|}
\hline \multicolumn{1}{|c|}{ Forces } & \multicolumn{1}{c|}{ Faiblesses } \\
\hline $\begin{array}{l}\text { Grand producteur de } \\
\text { coton et d'autres fibres } \\
\text { synthétiques }\end{array}$ & $\begin{array}{l}\text { Mauvaise qualité de la matière } \\
\text { première }\end{array}$ \\
\hline $\begin{array}{l}\text { Secteur textile } \\
\text { industrialisé }\end{array}$ & $\begin{array}{l}\text { Approvisionnement interne } \\
\text { insuffisant }\end{array}$ \\
\hline $\begin{array}{l}\text { Elimination des tarifs } \\
\text { douaniers d'importation }\end{array}$ & Risque pays élevé \\
\hline $\begin{array}{l}\text { Infrastructure de } \\
\text { transport interne } \\
\text { développée }\end{array}$ & $\begin{array}{l}\text { (triangle Sao Paulo, Belo } \\
\text { Horizonte, Rio de Janeiro) }\end{array}$ \\
\hline
\end{tabular}

Source. Elaboration des auteurs.

L'Argentine a reproduit, à plus petite échelle, le processus d'internalisation de l'offre de textiles et de vêtements du Brésil. Le pays produit aussi bien des fibres naturelles qu'artificielles. C'est un producteur de laine et de coton destinés à l'exportation même si un certain pourcentage de la production est absorbé par le marché intérieur et l'industrie textile. Le coton est la principale matière première utilisée mais il existe aussi des usines de fibres synthétiques, cellulosiques et acryliques. 
Les activités de filage, tissage et fabrication de vêtement représentent environ $60 \%$ de la valeur ajoutée du secteur textile. L'activité de tissage est la plus importante, elle représente $1 / 3 \mathrm{du}$ produit manufacturier textile. Les principaux produits textiles argentins sont: le prêt-à-porter (26\%), les sous-vêtements (19\%), et les tenues habillées pour hommes $(12 \%)$.

Le Paraguay possède une forte production de fibres de coton. Cependant l'activité de transformation est réduite.

L'Uruguay est un grand producteur de laine destinée à l'exportation (à peine 5\% est consommée par l'industrie textile locale). L'industrie de teinture de laine est particulièrement développée. Il existe une entreprise fabricant des fibres synthétiques (acétate, polyester). En Uruguay, il existe deux chaînes importantes de production textile: celle de l'industrie du coton et des tissus synthétiques, et celle des textiles en laine. Par ailleurs, l'abondante disponibilité de cuir a permis le développement de la fabrication de vêtement en cuir. L'industrie du vêtement en Uruguay est basée sur la fabrication de jeans, du prêt-àporter en toile, de la fabrication de tissus de pointe.

Avec l'ouverture commerciale des années 90, on a assisté dans tous les pays du MERCOSUR à un processus de désintégration vertical des chaînes de production textiles et vestimentaires. Une grande proportion de matières premières et biens intermédiaires ont commencé à être introduits par des producteurs étrangers remplaçant les producteurs locaux. La production du Mercosur est de plus de 4700 millions de vêtements par an. Plus de 80\% de cette production est réalisée au Brésil. La production de fibres naturelles, artificielles et synthétiques est destinée exclusivement à l'approvisionnement du marché textile local, ce qui s'explique par la dimension du marché interne brésilien et un niveau élevé de protectionnisme à travers des barrières douanières.

Les points forts du MERCOSUR se concentrent sur les "conditions sectorielles", comme le coût de la main d'œuvre, le coût des matières premières, mais aussi par rapport aux industries présentes sur ce marché et à l'évolution positive de la demande domestique.
Le Mexique: Malgré un contexte difficile lié à la forte concurrence des produits asiatiques, le secteur textile mexicain continue de se moderniser, et notamment dans les activités de teinture, de finition, de métiers à tisser, etc. La plupart des grandes entreprises mexicaines sont intégrées verticalement et effectuent elles-mêmes les différentes étapes de transformation depuis la filature jusqu'à l'ennoblissement des tissus. Parmi les principales sociétés mexicaines du secteur de l'habillement on trouve : Ferrioni, Levi's, Playtex et Hanes. La marque distributeur de Carrefour, «Tex», est également de fabrication mexicaine.

\subsection{Renforcer la position commerciale : développement de la notoriété et de l'image de marque}

Il n'existe que 12 magasins Zara au Brésil. Il y a donc des opportunités de développement sur ce marché qui passent par le développement d'une meilleure notoriété de ZARA et par le renforcement du positionnement commercial (avec les Mall Zara).

En Amérique latine, le positionnement haut de gamme de Zara se retrouve donc en concurrence avec Mango, Benetton, Chevignon, Esprit Mexx... En revanche, en Europe, la marque se positionne comme du «moyenne gamme». Il serait donc intéressant de revoir toute la stratégie marketing en réorientant la publicité et la promotion vers un secteur haut de gamme.

\subsection{La création de petites boutiques Zara: une alternative au concept de mégastore}

Il pourrait être envisageable de créer de plus petits magasins dans un certain nombre de villes intermédiaires d'Amérique Latine. Cela pourrait être envisagé comme une stratégie de pérennisation. Zara améliore sa notoriété, le groupe bénéficie de la bonne image de l'Europe, de la tradition de mode, de style avant- gardiste, et le concept de magasins à prix abordables est porteur. Mais comme on l'a précisé, ce système semble avoir atteint ses limites en Amérique Latine tant du point de vue commercial que de l'organisation productive. 
Comme alternative au concept de mégastore, on peut effectivement réfléchir à de petites boutiques en centreville. En marge de ce concept, il serait possible d'envisager une segmentation plus forte de Zara avec la création de petites boutiques spécialisées dans la mode masculine, féminine ou pour enfant et inclure dans ce concept les autres entités du groupe Inditex, de façon directe ou indirecte (magasin Zara pour les jeunes/adolescents avec les éléments que l'on retrouve dans la boutique Straddivarius, etc). En terme purement commercial, cette alternative pourrait donc constituer un élément clé dans la stratégie de développement de Zara en Amérique Latine.

\subsection{La création d'un « Mall Zara " : un autre concept innovant pour Zara}

Le terme de «Mall Zara » est inadéquat puisqu'il s'agit en fait d'un « Mall Inditex ». En effet, envisager un grand bâtiment regroupant tous les magasins du groupe Inditex avec un pôle habillement (Zara Homme, Femme, Enfant) ainsi que les autres boutiques, ou développer éventuellement, dans un second temps, de nouveaux magasins, cela pourrait se faire dans les grandes mégalopoles Latino-Américaines pour commencer. Il faut dire que ce projet a déjà été proposé en Espagne et en France.

Ce sont donc les alternatives qui pourraient être envisagées pour relancer le développement commercial du groupe Zara. Comme nous l'avons évoqué précédemment, il pourrait y avoir une autre alternative pour Zara, à savoir celle de profiter des possibilités de production offertes par le marché chinois. Cette alternative pourrait permettre à Zara d'envisager de nouvelles perspectives de développement. 


\section{LE DÉVELOPPEMENT EXPONENTIEL CHINOIS LAISSE DE CÔTÉ L'AMÉRIQUE LATINE}

$\mathbf{P}$ our le développement de Zara en Chine, il faut s'intéresser à l'environnement du pays afin de pouvoir s'adapter et permettre une gestion logistique efficiente et une maîtrise des coûts. En prenant connaissance du contexte chinois, Zara pourra déterminer sa position et développer une stratégie efficace dirigée aux marchés du prêt-à-porter latino-américains.

En prenant en compte la position de Zara sur le marché européen, il semble opportun de développer le même modèle de production en Chine, avec des outils et des modalités de travail permettant de garantir une qualité de production équivalente et à moindre coût. Les multiples accords que la Chine vient de réaliser avec le MERCOSUR et la Communauté Andine, sur l'ouverture de son marché laisse penser que les droits d'importation des pays Latinoaméricains seront revus à la baisse.
Les nouveaux avantages qui émergent en termes de politique commerciale pourraient être avantageux pour la mise en place de nouvelles stratégies de production qui impliqueraient cependant des changements drastiques dans les infrastructures logistiques et de distribution. Cela pourrait constituer une réponse à une distribution plus efficiente en Amérique Latine et dans le monde. Coûts logistiques réduits, forte rotation des voies maritimes et aériennes, spécialisation des chaînes de production textile, main d'œuvre bon marché et savoir-faire dans la confection, voici les principaux atouts qui font de la Chine une des futures plateformes logistiques internationales.

A la lumière de cette analyse, il peut être intéressant de voir quelles peuvent être les forces et les faiblesses, les opportunités et les contraintes d'implantation d'une unité productive pour Zara et ce dans une perspective de distribution sur le marché Latino-Américain.

Tableau 4. Analyse stratégique d'une implantation productive en Chine

\begin{tabular}{|l|l|}
\hline \multicolumn{1}{|c|}{ Forces } & \multicolumn{1}{|c|}{ Faiblesses } \\
\hline $\begin{array}{l}\text { - Expertise dans la production à grande échelle } \\
\text { - Organisation intrinsèque de la production } \\
\text { (renouvellement régulier des modèles, rotation } \\
\text { des patrons,...) } \\
\text { - Production similaire pour la majorité des pays : } \\
\begin{array}{l}\text { expertise et possibilité } \\
\text { - Risque pays faible }\end{array}\end{array}$ & $\begin{array}{l}\text { - Faible connaissance dans la production en Asie. } \\
\text { - Investissement lourds en unité de production. } \\
\text { - Changement fort dans la politique traditionnelle de Zara. } \\
\text { - Long délai de transport maritime (Pékin -Santos: environ 30 } \\
\text { jours) } \\
\text { - Coût de transport aérien élevé }\end{array}$ \\
\hline \multicolumn{1}{|c|}{ Opportunités } & \\
\hline $\begin{array}{l}\text { - Coût de la main d'œuvre } \\
\text { - Coût / approvisionnement en matière première } \\
\text { - Rayonnement productif sur tous les pays } \\
\text { d'implantation de Zara. }\end{array}$ & $\begin{array}{l}\text { - Contraintes } \\
\text { règlementaires,...) } \\
\text {-Fortcontrôle dela Chine(perted'autonomie etd'indépendance). } \\
\text { - Perception depuis la Corogne. }\end{array}$ \\
\hline
\end{tabular}

\section{Source. Elaboration des auteurs.}


Ce tableau permet de visualiser les grands enjeux que pourraient représenter une implantation productive de Zara en Chine. Cependant, et avant d'envisager formellement cette possibilité, il existe des prérequis dont Zara doit impérativement tenir compte pour réussir une implantation de cette nature.

- Suivi de la qualité. Le maintien de la qualité de la marque Zara dépendra du type d'entreprise qui réalisera le processus de production et du type de joint-venture effectué (impératifs de suivi des standards internationaux de production, notamment avec les normes ISO). Zara devra cependant garder un contrôle substantiel sur la production.

- Développement commercial. En développant ses activités commerciales sur le marché Latino-américain et souhaitant créer une entité productive, il s'agira également de définir quels seront les objectifs de la marque.

Cette partie comprend nécessairement une croissance des magasins présents sur le continent car, excepté le Mexique et 56 magasins, le Brésil et 39 magasins, la Colombie et 11 magasins et l'Argentine et 10 magasins, les autres pays connaissent peu la marque et afin de rentabiliser au maximum une unité de production lourde en Asie, il serait indispensable de garantir de nouveaux et significatifs débouchés commerciaux. Pour cette raison, la marque Zara doit développer son image afin d'être mieux connue et reconnue. Cela peut être réalisé par le biais d'une campagne de communication et de publicité, de défilés.

\section{1 ¿Quelles modalités d'implantation?}

- Alliances stratégiques pour faciliter l'exportation de produits et de marchandises. Les précédents positifs en Chine impliquent généralement des joint-ventures ou des investissements partagés pour faciliter la distribution de produits.

- Profiter des avantages douaniers pour les produits négociés entre la Chine, le MERCOSUR et la Communauté Andine des Nations (CAN).

- Réorganisation de la logistique globale de Zara afin d'intégrer ce nouveau flux de production dans son flux existant, notamment depuis La Corogne, et la construction d'un nouveau réseau logistique depuis la Chine. 


\section{CONCLUSIONS}

Il est donc indispensable que Zara parvienne à conjuguer développement commercial et intensification productive. Ce développement commercial passera par une nouvelle réflexion sur les modalités de développement pour 1'Amérique Latine (nouvelles structures) et sur une refonte de la production afin de pouvoir accompagner au mieux cet essor commercial naissant.

En Asie, la production serait possible à condition que la main d'œuvre soit encore moins chère. Notons tout de même que la logique de localisation de la production textile ne suit pas exactement la répartition des bas salaires dans le monde. Elle est soumise à une contrainte de proximité imposée par le cycle court des produits et à la réactivité qu'il faut conserver dans le domaine de la mode.

L'Amérique latine reste donc une région clef pour le groupe Inditex et Zara qui possèdent la majorité de leurs magasins hors d'Europe. Cependant, comme nous avons pu le voir, de nombreux défis se posent:

- Défi logistique: les infrastructures de transport entre pays empêchent d'envisager un système productif propre à l'Amérique latine qui serait trop onéreux et qui n'apporterait pas d'économies d'échelles géographiques.

- Défi commercial: En Amérique latine, Zara est une marque accessible et innovante. Elle subit cependant la concurrence de marques positionnées en haut de gamme qui profitent d'une classe moyenne de plus en plus aisée (plus ou moins vaste selon les pays). Il pourrait donc être intéressant de revoir la stratégie marketing de Zara en améliorant et en renforçant la publicité et la promotion des produits.

Avec les potentialités offertes par le marché chinois - et asiatique en général-il semble intéressant de réfléchir à une réorganisation globale de l'activité productive du groupe. En effet, même si Inditex s'est déjà commercialement implanté en Asie, il pourrait être judicieux de réfléchir à un développement productif dans cette région. Cela serait certes un changement radical de la politique traditionnelle $\mathrm{du}$ groupe sur ces questions (privilégiant le maintien de l'activité à La Corogne, en Espagne), mais il serait dangereux d'écarter ce genre d'hypothèses.

Deux recommandations peuvent être faites:

- Privilégier une nouvelle politique commerciale en Amérique latine (Mall Zara,...) afin d'impulser la demande et créer de nouveaux besoins.

- D'accompagnemer cette augmentation de l'offre par une implantation productive en Chine (qui permettrait d'obtenir des coûts réduits) tout en réfléchissant au système logistique global du groupe. 


\section{REFÉRENCES}

Badia, E. (2008) ZARA y sus hermanas, LIB editorial empresarial, Madrid, España.

Barreiro, A. (2008) Hacia un nuevo sistema de la moda: El modelo Zara. Revista Internacional de Sociología (RIS), Vol. LXVI, n ${ }^{\circ}$ 51, Septiembre-Diciembre, 105-122.

Belmiro do Nascimento, J. et al (2010) Internacionalizacion en la industria de la moda: El caso Zara. Revista Galega de Economía, 19, (2),p 1-23

Blanco, X., Salgado, J. (2004) Amancio Ortega: de cero a Zara, el primer libro de investigación sobre el imperio Inditex, Editorial. La Esfera de los Libros.

Breuzard, J., Frometin, D. (2005) Reussir la logistique des activites de services. Les editions DEMOS, Paris.

Castro M., González V. Los efectos de la orientaciónal mercado en el desarrollo de ventajas competitivas sostenibles y en los comportamientos empresariales- El caso Zara-Inditex.

Carballo, R. (2004) En la espiral de la innovación: modelo banchmarking y experiencias de empresas innovadoras en España. (Inditex, Ingenio, Irizar, MRW, e-Human@, Aldebaran Innovation, Pharma-Mar, Tecasa), Editorial Díaz de Santos.

Castellano, J. (2002) El proceso de internacionalización de INDITEX, ICE Empresas Multinaciones Españolas, No. 799 (p.209-217).

Fábrega, F. (2004) Zara, Editorial Claves de Gestión.

INDITEX, Informes anuales 2000-2001-2002-2003-2004-2005-2006-2007-2008-2009-2010-2011-2012 www.inditex.es.

Kotler, P. (2007) Marketing Versión para Latino América, décimo primera edición, Pearson Prentice Hall. p 196-212.

Lee K. (2005) Marketing internacional: Teorías y casos. Cengage Learnig, México. p 27-28.

Lopez, C.; Fan Y. (2009) Internationalisation of the spanish fashion brand ZARA. Journal of Fashion Marketing and Management 13(2), p 279-296.

Mazzanti, M.; Montresor S. (2008) Outsourcing, Delocalization and Firm Organization: Transaction Costs vs. Industrial Relations in a Local Production System of Emilia-Romagna, DRUID Working Paper No. 08-08.

Monllor, C. (2001) Zarápolis, la historia secreta de un imperio de la moda, Ediciones del Bronce.

O’Shea, C. (2008) Así es Amancio Ortega, el hombre que creó Zara, Editorial La Esfera de los Libros. 
Rapport mundial sur les salaires 2012/2013. Salaires et croissance équitable, (2012) Conditions of Work and Employment Branch(TRAVAIL), Organisation International du Travail (OIT).

Reuters, (2012) Análisis: diferencias políticas y protestas nublan las inversiones en A. Latina. Revista América Económica, [consultado el 16/07/2013] Disponible en http://www.americaeconomia.com/economia-mercados/finanzas/analisisdiferencias-politicas-y-protestas-nublan-inversiones-en-alatina

Tungate, Mark, Page Kogan (2005) Fashion Brands: Branding Style from Armani to Zara.

Ugarte, J. "México, la joya de Zara en América” CNN Expancio.com [en línea]

[Consultado el 16 de Julio de 2013]. Disponible en http://www.cnnexpansion.com/negocios/2011/01/26/mexico-la-joyade-zara-en-america 